\title{
Coefficient Bounds for Some Families of Starlike and Convex Functions of Reciprocal Order
}

\author{
Muhammad Arif,, ${ }^{1}$ Maslina Darus, ${ }^{2}$ Mohsan Raza, ${ }^{3}$ and Qaiser Khan ${ }^{1}$ \\ ${ }^{1}$ Department of Mathematics, Abdul Wali Khan University Mardan, Mardan, Khyber Pakhtunkhwa 23200, Pakistan \\ ${ }^{2}$ School of Mathematical Sciences, Faculty of Science and Technology, Universiti Kebangsaan Malaysia, 43600 Bangi, \\ Selangor, Malaysia \\ ${ }^{3}$ Department of Mathematics, Government College University, Faisalabad 38000, Pakistan
}

Correspondence should be addressed to Muhammad Arif; marifmaths@yahoo.com

Received 31 May 2014; Revised 7 September 2014; Accepted 12 October 2014; Published 24 November 2014

Academic Editor: Minghe Sun

Copyright (C) 2014 Muhammad Arif et al. This is an open access article distributed under the Creative Commons Attribution License, which permits unrestricted use, distribution, and reproduction in any medium, provided the original work is properly cited.

The aim of the present paper is to investigate coefficient estimates, Fekete-Szegö inequality, and upper bound of third Hankel determinant for some families of starlike and convex functions of reciprocal order.

\section{Introduction}

Let $\mathscr{A}$ denote the class of functions $f(z)$ which are analytic in the open unit disk $\mathbb{U}=\{z \in \mathbb{C}:|z|<1\}$ and normalized by

$$
f(z)=z+\sum_{k=2}^{\infty} a_{k} z^{k}, \quad(z \in \mathbb{U})
$$

Also let $\mathcal{S}^{*}(\alpha)$ and $\mathscr{K}(\alpha)$ denote the usual classes of starlike and convex functions of order $\alpha, 0 \leq \alpha<1$, respectively. In 1975 , Silverman [1] proved that $f(z) \in \mathcal{S}^{*}(\alpha)$ if it satisfies the condition

$$
\left|\frac{z f^{\prime}(z)}{f(z)}-1\right|<1-\alpha, \quad(z \in \mathbb{U})
$$

Geometrical meaning of inequality (2) is that $z f^{\prime}(z) / f(z)$ maps $\mathbb{U}$ onto the interior of the circle with center at 1 and radius $1-\alpha$.
By $\mathcal{S}_{*}(\alpha)$ and $\mathscr{K}_{*}(\alpha)$, we mean the classes of starlike and convex functions of reciprocal order $\alpha, 0 \leq \alpha<1$ which are defined, respectively, by

$$
\begin{gathered}
\mathcal{S}_{*}(\alpha)=\left\{f(z) \in \mathscr{A}: \operatorname{Re} \frac{f(z)}{z f^{\prime}(z)}>\alpha,(z \in \mathbb{U})\right\}, \\
\mathscr{K}_{*}(\alpha)=\left\{f(z) \in \mathscr{A}: \operatorname{Re} \frac{f^{\prime}(z)}{z f^{\prime \prime}(z)+f^{\prime}(z)}>\alpha,(z \in \mathbb{U})\right\} .
\end{gathered}
$$

Recently in 2008, Nunokawa and his coauthors [2] improved inequality (2) for the class $\delta_{*}(\alpha)$ and they proved that, for $f(z) \in \mathcal{S}_{*}(\alpha), 0<\alpha<1 / 2$, if and only if the following inequality holds:

$$
\left|\frac{z f^{\prime}(z)}{f(z)}-\frac{1}{2 \alpha}\right|<\frac{1}{2 \alpha}, \quad(z \in \mathbb{U}) .
$$

In view of these results we now define the following subclass of analytic functions of reciprocal order and investigate its various properties. 
Definition 1. A function $f(z) \in \mathscr{A}$ is said to be in the class $\mathscr{L}(\lambda, \gamma)$, with $\gamma \in \mathbb{C} \backslash\{0\}$ and $\lambda \in[0,1]$, if it satisfies the inequality

$$
\operatorname{Re}\left(1+\frac{1}{\gamma}\left(\frac{F_{\lambda}(z)}{z F_{\lambda}^{\prime}(z)}-1\right)\right)>0
$$

where

$$
F_{\lambda}(z)=(1-\lambda) f(z)+\lambda z f^{\prime}(z) .
$$

Example 2. Let us define the functions $F_{\lambda}(z)$ by

$$
F_{\lambda}(z)=\frac{z}{(1+(2 \gamma-1) z)^{2 \gamma /(2 \gamma-1)}} .
$$

This implies that

$$
\frac{z F_{\lambda}^{\prime}(z)}{F_{\lambda}(z)}=\frac{1-z}{1+(2 \gamma-1) z}
$$

Hence

$$
1+\frac{1}{\gamma}\left(\frac{F_{\lambda}(z)}{z F_{\lambda}^{\prime}(z)}-1\right)=\frac{1+z}{1-z}
$$

and this further implies that

$$
\operatorname{Re}\left(1+\frac{1}{\gamma}\left(\frac{F_{\lambda}(z)}{z F_{\lambda}^{\prime}(z)}-1\right)\right)=\operatorname{Re} \frac{1+z}{1-z}>0, \quad(z \in \mathbb{U}) .
$$

The $q$ th Hankel determinant $H_{q}(n), q \geq 1, n \geq 1$, for a function $f(z) \in \mathscr{A}$ is studied by Noonan and Thomas [3] as

$$
H_{q}(n)=\left|\begin{array}{cccc}
a_{n} & a_{n+1} & \cdots & a_{n+q-1} \\
a_{n+1} & a_{n+2} & \cdots & a_{n+q} \\
\vdots & \vdots & \cdots & \vdots \\
a_{n+q-1} & a_{n+q} & \cdots & a_{n+2 q-2}
\end{array}\right| .
$$

In literature many authors have studied the determinant $H_{q}(n)$. For example, Arif et al. $[4,5]$ studied the $q$ th Hankel determinant for some subclasses of analytic functions. Hankel determinant of exponential polynomials is obtained by Ehrenborg in [6]. The Hankel transform of an integer sequence and some of its properties were discussed by Layman [7]. It is well known that the Fekete-Szegö functional $\left|a_{3}-a_{2}^{2}\right|$ is $H_{2}(1)$. Fekete-Szego" then further generalized the estimate $\left|a_{3}-\lambda a_{2}^{2}\right|$ with $\lambda$ real and $f(z) \in \mathcal{S}$. Moreover, we also know that the functional $\left|a_{2} a_{4}-a_{3}^{2}\right|$ is equivalent to $H_{2}(2)$. The sharp upper bounds of the second Hankel determinant for the familiar classes of starlike and convex functions were studied by Janteng et al. [8]; that is, for $f(z) \in \mathcal{S}^{*}$ and $f(z) \in \mathscr{C}$, they obtained $\left|a_{2} a_{4}-a_{3}^{2}\right| \leq 1$ and $8\left|a_{2} a_{4}-a_{3}^{2}\right| \leq 1$, respectively. In 2007, Babalola [9] considered the third Hankel determinant $H_{3}(1)$ and obtained the upper bound of the well-known classes of bounded-turning, starlike and convex functions. In 2013 Raza and Malik [10] studied the Hankel third determinant related with lemniscate of Bernoulli. In the present investigation, we study the upper bound of $H_{3}(1)$ for a subclass of analytic functions of reciprocal order by using Toeplitz determinants.
In this paper we study some useful results including coefficient estimates, Fekete-Szegő inequality, and upper bound of third Hankel determinant for the functions belonging to the class $\mathscr{L}(\lambda, \gamma)$.

Throughout in this paper we assume that $\gamma \in \mathbb{C} \backslash\{0\}$ and $\lambda \in[0,1]$ unless otherwise stated.

For our results we will need the following Lemmas.

Lemma 3 (see [11]). If $q(z)$ is a function with $\operatorname{Re} q(z)>0$ and is of the form

$$
q(z)=1+c_{1} z+c_{2} z^{2}+\cdots
$$

then

$$
\left|c_{n}\right| \leq 2, \quad \text { for } n \geq 1 \text {. }
$$

Lemma 4 (see [12]). If $q(z)$ is of the form (12) with positive real part, then the following sharp estimate holds:

$$
\left|c_{2}-v c_{1}^{2}\right| \leq 2 \max \{1,|2 v-1|\}, \quad \text { for all } v \in \mathbb{C} .
$$

Lemma 5 (see [13]). If $q(z)$ is of the form (12) with positive real part, then

$$
\begin{gathered}
2 c_{2}=c_{1}^{2}+x\left(4-c_{1}^{2}\right), \\
4 c_{3}=c_{1}^{3}+2\left(4-c_{1}^{2}\right) c_{1} x-c_{1}\left(4-c_{1}^{2}\right) x^{2} \\
+2\left(4-c_{1}^{2}\right)\left(1-|x|^{2}\right) z,
\end{gathered}
$$

for some $x, z$ with $|x| \leq 1$ and $|z| \leq 1$.

\section{Some Properties of the Class $\mathscr{L}(\lambda, \gamma)$}

Theorem 6. Let $f(z) \in \mathscr{L}(\lambda, \gamma)$. Then

$$
\left|a_{2}\right| \leq \frac{2|\gamma|}{1+\lambda}
$$

and for all $n=3,4,5, \ldots$

$$
\left|a_{n}\right| \leq \frac{2|\gamma|}{(n-1)(1+\lambda(n-1))} \prod_{k=2}^{n-1}\left(1+\frac{2|\gamma| k}{k-1}\right) .
$$

Proof. Let us define the function $q(z)$ by

$$
q(z)=1+\frac{1}{\gamma}\left(\frac{F_{\lambda}(z)}{z F_{\lambda}^{\prime}(z)}-1\right)
$$

where $F_{\lambda}(z)$ is given by (6) with

$$
F_{\lambda}(z)=z+\sum_{k=2}^{\infty}[1+\lambda(k-1)] a_{k} z^{k}
$$

and $q(z)$ is analytic in $\mathbb{U}$ with $q(0)=1, \operatorname{Re} q(z)>0$.

Now using (1) and (12), we have

$$
z+\sum_{k=2}^{\infty} A_{k} z^{k}=\left[1+\gamma\left(\sum_{k=1}^{\infty} c_{k} z^{k}\right)\right]\left(z+\sum_{k=2}^{\infty} k A_{k} z^{k}\right),
$$


where

$$
A_{k}=[1+\lambda(k-1)] a_{k} .
$$

Comparing coefficient of like power of $z^{n}$, we obtain

$$
(1-n) A_{n}=\gamma\left\{c_{n-1}+2 A_{2} c_{n-2}+\cdots+(n-1) A_{n-1} c_{1}\right\} .
$$

Using triangle inequality and Lemma 3 , we get

$$
\left|(1-n) A_{n}\right| \leq 2|\gamma|\left\{1+2\left|A_{2}\right|+\cdots+(n-1)\left|A_{n-1}\right|\right\} .
$$

For $n=2$ and $n=3$ in (23), we easily obtain that

$$
\left|a_{2}\right| \leq \frac{2|\gamma|}{1+\lambda}, \quad\left|a_{3}\right| \leq \frac{|\gamma|(1+4|\gamma|)}{1+2 \lambda} .
$$

Making $n=4$ in (23), we see that

$$
2\left|A_{3}\right| \leq 2|\gamma|\left(1+2\left|A_{2}\right|\right) \leq 2|\gamma|\left\{1+\frac{4|\gamma|(1+2 \lambda)}{1+\lambda}\right\} ;
$$

equivalently, we have

$$
\left|a_{4}\right| \leq \frac{2|\gamma|(1+3|\gamma|)(1+4|\gamma|)}{3(1+3 \lambda)} .
$$

Using the principal of mathematical induction, we obtain

$$
\left|A_{n}\right| \leq \frac{2|\gamma|}{(n-1)} \prod_{k=2}^{n-1}\left(1+\frac{2|\gamma| k}{k-1}\right) .
$$

Now from the use of relation (21), we obtain the required result.

If we take $\lambda=0$ and $\gamma=1-\alpha$, we get the following result.

Corollary 7 (see [14]). Let $f(z) \in \mathcal{S}_{*}(\alpha)$. Then, for $n=$ $3,4,5, \ldots$, one has

$$
\left|a_{n}\right| \leq \frac{2(1-\alpha)}{(n-1)} \prod_{k=2}^{n-1}\left(1+\frac{2(1-\alpha) k}{k-1}\right),
$$

with $\left|a_{2}\right| \leq 2(1-\alpha)$.

Making $\lambda=1$ and $\gamma=1-\alpha$, we get the following result.

Corollary 8 (see [14]). Let $f(z) \in \mathscr{K}_{*}(\alpha)$. Then, for $n=$ $3,4,5, \ldots$, one has

$$
\left|a_{n}\right| \leq \frac{2(1-\alpha)}{n(n-1)} \prod_{k=2}^{n-1}\left(1+\frac{2(1-\alpha) k}{k-1}\right)
$$

with $\left|a_{2}\right| \leq(1-\alpha)$.
Theorem 9. Let $f(z) \in \mathscr{L}(\lambda, \gamma)$ and be of the form (1). Then

$$
\left|a_{3}-\mu a_{2}^{2}\right| \leq \frac{|\gamma|}{(1+2 \lambda)} \max \{1,|2 v-1|\},
$$

where

$$
\nu=2 \gamma(1+2 \lambda)\left(\frac{1}{1+2 \lambda}-\frac{\mu}{(1+\lambda)^{2}}\right) .
$$

Proof. Let $f(z) \in \mathscr{L}(\lambda, \gamma)$. Then from (22) we have

$$
a_{2}=\frac{-\gamma c_{1}}{(1+\lambda)}, \quad a_{3}=\frac{-\gamma}{2(1+2 \lambda)}\left(c_{2}-2 \gamma c_{1}^{2}\right) .
$$

We now consider

$$
\begin{aligned}
& \left|a_{3}-\mu a_{2}^{2}\right| \\
& =\frac{|\gamma|}{2(1+2 \lambda)}\left|c_{2}-2 \gamma(1+2 \lambda)\left(\frac{1}{1+2 \lambda}-\frac{\mu}{(1+\lambda)^{2}}\right) c_{1}^{2}\right| .
\end{aligned}
$$

Using Lemma 4, we obtain

$$
\left|a_{3}-\mu a_{2}^{2}\right| \leq \frac{|\gamma|}{(1+2 \lambda)} \max \{1,|2 v-1|\},
$$

where $v$ is given by (31).

Putting $\mu=1$, we obtain the following result.

Corollary 10. Let $f(z) \in \mathscr{L}(\lambda, \gamma)$. Then

$$
\left|a_{3}-a_{2}^{2}\right| \leq \frac{|\gamma|}{(1+2 \lambda)} .
$$

Theorem 11. Let $f(z) \in \mathscr{L}(\lambda, \gamma)$ and be of the form (1). Then

$$
\begin{aligned}
& \left|a_{2} a_{4}-a_{3}^{2}\right| \\
& \leq\left[\frac{7+28 \lambda+25 \lambda^{2}+4\left(1+4 \lambda+10 \lambda^{2}\right)|\gamma|+48 \lambda^{2}|\gamma|^{2}}{3(1+2 \lambda)^{2}\left(1+4 \lambda+3 \lambda^{2}\right)}\right] \\
& \quad \times|\gamma|^{2} .
\end{aligned}
$$

Proof. Let $f(z) \in \mathscr{L}(\lambda, \gamma)$. Then, from (22), we have

$$
\begin{aligned}
& a_{2}=\frac{-\gamma c_{1}}{(1+\lambda)}, \\
& a_{3}=\frac{-\gamma}{2(1+2 \lambda)}\left(c_{2}-2 \gamma c_{1}^{2}\right), \\
& a_{4}=\frac{-\gamma}{3(1+3 \lambda)}\left(c_{3}-\frac{7 \gamma}{2} c_{1} c_{2}+3 \gamma^{2} c_{1}^{3}\right) .
\end{aligned}
$$




\section{Consider}

$$
\begin{aligned}
& \left|a_{2} a_{4}-a_{3}^{2}\right| \\
& =\mid \frac{\gamma^{2}}{12(1+\lambda)(1+2 \lambda)^{2}(1+3 \lambda)} \\
& \times\left(4(1+2 \lambda)^{2} c_{1} c_{3}-2 \gamma\left(1+4 \lambda+10 \lambda^{2}\right) c_{1}^{2} c_{2}\right. \\
& \left.+12 \gamma^{2} \lambda^{2} c_{1}^{4}-3\left(1+4 \lambda+3 \lambda^{2}\right) c_{2}^{2}\right) \mid
\end{aligned}
$$

Now using values of $c_{2}$ and $c_{3}$ from Lemma 5 , we obtain

$$
\begin{aligned}
& \left|a_{2} a_{4}-a_{3}^{2}\right| \\
& =\frac{|\gamma|^{2}}{12(1+\lambda)(1+2 \lambda)^{2}(1+3 \lambda)} \\
& \times \mid\left\{(1+2 \lambda)^{2}-\gamma\left(1+4 \lambda+10 \lambda^{2}\right)\right. \\
& \left.\quad+12 \gamma^{2} \lambda^{2}-\frac{3}{4}\left(1+4 \lambda+3 \lambda^{2}\right)\right\} c_{1}^{4} \\
& \quad+\left\{2(1+2 \lambda)^{2}-\gamma\left(1+4 \lambda+10 \lambda^{2}\right)-\frac{3}{2}\left(1+4 \lambda+3 \lambda^{2}\right)\right\} \\
& \quad \times\left(4-c_{1}^{2}\right) c_{1}^{2} x \\
& \quad-\left\{(1+2 \lambda)^{2} c_{1}^{2}+\frac{3}{4}\left(1+4 \lambda+3 \lambda^{2}\right)\left(4-c_{1}^{2}\right)\right\} \\
& \quad \times\left(4-c_{1}^{2}\right) x^{2}+2 c_{1}(1+2 \lambda)^{2}\left(4-c_{1}^{2}\right)\left(1-|x|^{2}\right) z \mid .
\end{aligned}
$$

Applying triangle inequality and replacing $c_{1}$ by $c,|x|$ by $\rho$, and $|z|$ by 1 , we get

$$
\begin{aligned}
& \left|a_{2} a_{4}-a_{3}^{2}\right| \quad \frac{|\gamma|^{2}}{12(1+\lambda)(1+2 \lambda)^{2}(1+3 \lambda)} \\
& \quad \times\left[\left\{(1+2 \lambda)^{2}+|\gamma|\left(1+4 \lambda+10 \lambda^{2}\right)\right.\right. \\
& \left.\quad+12|\gamma|^{2} \lambda^{2}+\frac{3}{4}\left(1+4 \lambda+3 \lambda^{2}\right)\right\} c^{4} \\
& \quad+\left\{2(1+2 \lambda)^{2}+|\gamma|\left(1+4 \lambda+10 \lambda^{2}\right)+\frac{3}{2}\left(1+4 \lambda+3 \lambda^{2}\right)\right\} \\
& \quad \times\left(4-c^{2}\right) c^{2} \rho \\
& \quad+\left\{(1+2 \lambda)^{2} c^{2}+\frac{3}{4}\left(1+4 \lambda+3 \lambda^{2}\right)\left(4-c^{2}\right)\right\} \\
& \left.\quad \times\left(4-c^{2}\right) \rho^{2}+2 c(1+2 \lambda)^{2}\left(4-c^{2}\right)\left(1-\rho^{2}\right)\right]=F(c, \rho) .
\end{aligned}
$$

Differentiating with respect to $\rho$, we get

$$
\begin{aligned}
& \frac{\partial F(c, \rho)}{\partial \rho} \\
& =\frac{|\gamma|^{2}}{12(1+\lambda)(1+2 \lambda)^{2}(1+3 \lambda)} \\
& \quad \times\left[\left\{2(1+2 \lambda)^{2}+|\gamma|\left(1+4 \lambda+10 \lambda^{2}\right)\right.\right. \\
& \left.\quad+\frac{3}{2}\left(1+4 \lambda+3 \lambda^{2}\right)\right\}\left(4-c^{2}\right) c^{2} \\
& +\left\{2(1+2 \lambda)^{2} c^{2}+\frac{3}{2}\left(1+4 \lambda+3 \lambda^{2}\right)\left(4-c^{2}\right)\right\} \\
& \left.\times\left(4-c^{2}\right) \rho-4 c(1+2 \lambda)^{2}\left(4-c^{2}\right) \rho\right] .
\end{aligned}
$$

Now since $\partial F(c, \rho) / \partial \rho>0$ for $c \in[0,2]$ and $\rho \in[0,1]$, maximum of $F(c, \rho)$ will exist at $\rho=1$ and let $F(c, 1)=G(c)$. Then

$$
\begin{aligned}
G(c)= & \frac{|\gamma|^{2}}{12(1+\lambda)(1+2 \lambda)^{2}(1+3 \lambda)} \\
& \times\left[\left\{(1+2 \lambda)^{2}+|\gamma|\left(1+4 \lambda+10 \lambda^{2}\right)\right.\right. \\
& \left.+12|\gamma|^{2} \lambda^{2}+\frac{3}{4}\left(1+4 \lambda+3 \lambda^{2}\right)\right\} c^{4} \\
& +\left\{2(1+2 \lambda)^{2}+|\gamma|\left(1+4 \lambda+10 \lambda^{2}\right)\right. \\
& +\left\{(1+2 \lambda)^{2} c^{2}+\frac{3}{4}\left(1+4 \lambda+3 \lambda^{2}\right)\left(4-c^{2}\right)\right\} \\
& \left.\times\left(4-c^{2}\right)\right] .
\end{aligned}
$$

Now by differentiating with respect to $c$, we obtain

$$
\begin{aligned}
G^{\prime}(c)= & \frac{|\gamma|^{2}}{12(1+\lambda)(1+2 \lambda)^{2}(1+3 \lambda)} \\
& \times\left[4 \left\{(1+2 \lambda)^{2}+|\gamma|\left(1+4 \lambda+10 \lambda^{2}\right)\right.\right. \\
& \left.+12|\gamma|^{2} \lambda^{2}+\frac{3}{4}\left(1+4 \lambda+3 \lambda^{2}\right)\right\} c^{3} \\
+ & \left\{2(1+2 \lambda)^{2}+|\gamma|\left(1+4 \lambda+10 \lambda^{2}\right)\right. \\
& \left.+\frac{3}{2}\left(1+4 \lambda+3 \lambda^{2}\right)\right\}\left(8 c-4 c^{3}\right)
\end{aligned}
$$




$$
\begin{aligned}
& +\left\{(1+2 \lambda)^{2}\left(8 c-4 c^{3}\right)\right. \\
& \left.\left.\quad-3\left(1+4 \lambda+3 \lambda^{2}\right)\left(4 c-c^{3}\right)\right\}\right] .
\end{aligned}
$$

Since $\partial G(c) / \partial c>0$ for $c \in[0,2], G(c)$ has a maximum value at $c=2$ and hence

$$
\begin{aligned}
& \left|a_{2} a_{4}-a_{3}^{2}\right| \\
& \leq\left[\frac{7+28 \lambda+25 \lambda^{2}+4\left(1+4 \lambda+10 \lambda^{2}\right)|\gamma|+48 \lambda^{2}|\gamma|^{2}}{3(1+2 \lambda)^{2}\left(1+4 \lambda+3 \lambda^{2}\right)}\right] \\
& \quad \times|\gamma|^{2} .
\end{aligned}
$$

Theorem 12. Let $f(z) \in \mathscr{L}(\lambda, \gamma)$ and be of the form (1). Then

$$
\left|a_{2} a_{3}-a_{4}\right| \leq \frac{2|\gamma|\left[(4|\gamma|+1)\left((6|\gamma|+2) \lambda^{2}+(3 \lambda+1)\right)\right]}{3(1+\lambda)(1+2 \lambda)(1+3 \lambda)}
$$

Proof. From (37), we can write

$$
\begin{aligned}
& \left|a_{2} a_{3}-a_{4}\right| \\
& =\left|\frac{12 \gamma^{2} \lambda^{2} c_{1}^{3}-2 \gamma\left(2+6 \lambda+7 \lambda^{2}\right) c_{1} c_{2}+2\left(1+3 \lambda+2 \lambda^{2}\right) c_{3}}{6(1+\lambda)(1+2 \lambda)(1+3 \lambda)}\right| \\
& \quad \times|\gamma| .
\end{aligned}
$$

Using Lemma 5 for the values of $c_{2}$ and $c_{3}$, we have

$$
\begin{aligned}
\mid a_{2} a_{3}- & a_{4} \mid \\
= & \frac{|\gamma|}{6(1+\lambda)(1+2 \lambda)(1+3 \lambda)} \\
& \times \mid \frac{1}{2}(4 \gamma-1)\left(2(3 \gamma-1) \lambda^{2}-(3 \lambda+1)\right) c_{1}^{3} \\
& +\left(\left(1+3 \lambda+2 \lambda^{2}\right)-\gamma\left(2+6 \lambda+7 \lambda^{2}\right)\right) \\
& \times\left(4-c_{1}^{2}\right) c_{1} x \\
& -\frac{1}{2}\left(1+3 \lambda+2 \lambda^{2}\right)\left(4-c_{1}^{2}\right) c_{1} x^{2} \\
& +\left(1+3 \lambda+2 \lambda^{2}\right)\left(4-c_{1}^{2}\right)\left(1-|x|^{2}\right) z \mid .
\end{aligned}
$$

Applying triangle inequality and then putting $|z|=1,|x|=\rho$, and $c_{1}=c$, we have

$$
\begin{aligned}
& \left|a_{2} a_{3}-a_{4}\right| \\
& \leq \frac{|\gamma|}{6(1+\lambda)(1+2 \lambda)(1+3 \lambda)} \\
& \quad \times\left[(4|\gamma|+1)\left((6|\gamma|+2) \lambda^{2}+(3 \lambda+1)\right) \frac{c^{3}}{2}\right. \\
& \quad+\left(\left(1+3 \lambda+2 \lambda^{2}\right)+|\gamma|\left(2+6 \lambda+7 \lambda^{2}\right)\right) \\
& \quad \times\left(4-c^{2}\right) c \rho+\frac{1}{2}\left(1+3 \lambda+2 \lambda^{2}\right)\left(4-c^{2}\right) c \rho^{2} \\
& \left.\quad+\left(1+3 \lambda+2 \lambda^{2}\right)\left(4-c^{2}\right)\left(1-\rho^{2}\right)\right]=F(c, \rho) .
\end{aligned}
$$

Now by using the same procedure as we did in the proof of Theorem 11, we obtain the required result.

Theorem 13. If $f(z) \in \mathscr{L}(\lambda, \gamma)$ and is of the form (1), then

$$
\begin{aligned}
& \left|H_{3}(1)\right| \\
& \leq\left[\frac{7+28 \lambda+25 \lambda^{2}+4\left(1+4 \lambda+10 \lambda^{2}\right)|\gamma|+48 \lambda^{2}|\gamma|^{2}}{3(1+2 \lambda)^{3}\left(1+4 \lambda+3 \lambda^{2}\right)}\right] \\
& \quad \times|\gamma|^{3}(1+4|\gamma|) \\
& \quad+\left[\frac{4\left[(4|\gamma|+1)^{2}\left((3|\gamma|+1) 2 \lambda^{2}+(3 \lambda+1)\right)\right]}{9(1+\lambda)(1+2 \lambda)(1+3 \lambda)^{2}}\right] \\
& \quad \times|\gamma|^{2}(1+3|\gamma|) \\
& \quad+\frac{(1+4|\gamma|)(1+3|\gamma|)(3+8|\gamma|)|\gamma|^{2}}{6(1+2 \lambda)(1+4 \lambda)} .
\end{aligned}
$$

Proof. Since

$$
\left|H_{3}(1)\right| \leq\left|a_{3}\right|\left|a_{2} a_{4}-a_{3}^{2}\right|+\left|a_{4}\right|\left|a_{2} a_{3}-a_{4}\right|+\left|a_{5}\right|\left|a_{1} a_{3}-a_{2}^{2}\right|,
$$

using Theorem 6, Corollary 10, and Theorems 11 and 12, we have

$$
\begin{aligned}
& \left|H_{3}(1)\right| \\
& \leq \frac{|\gamma|(1+4|\gamma|)}{(1+2 \lambda)} \\
& \quad \times\left[\frac{7+28 \lambda+25 \lambda^{2}+4\left(1+4 \lambda+10 \lambda^{2}\right)|\gamma|+48 \lambda^{2}|\gamma|^{2}}{3(1+2 \lambda)^{2}\left(1+4 \lambda+3 \lambda^{2}\right)}\right]
\end{aligned}
$$




$$
\begin{aligned}
& \times|\gamma|^{2}+\frac{2|\gamma|(1+4|\gamma|)(1+3|\gamma|)}{3(1+3 \lambda)} \\
& \times\left[\frac{2|\gamma|\left[(4|\gamma|+1)\left((3|\gamma|+1) 2 \lambda^{2}+(3 \lambda+1)\right)\right]}{3(1+\lambda)(1+2 \lambda)(1+3 \lambda)}\right] \\
& +\frac{|\gamma|(1+4|\gamma|)(1+3|\gamma|)(3+8|\gamma|)}{6(1+4 \lambda)} \frac{|\gamma|}{(1+2 \lambda)} \\
& =\left[\frac{7+28 \lambda+25 \lambda^{2}+4\left(1+4 \lambda+10 \lambda^{2}\right)|\gamma|+48 \lambda^{2}|\gamma|^{2}}{3(1+2 \lambda)^{3}\left(1+4 \lambda+3 \lambda^{2}\right)}\right] \\
& \times|\gamma|^{3}(1+4|\gamma|) \\
& +\left[\frac{4\left[(4|\gamma|+1)^{2}\left((3|\gamma|+1) 2 \lambda^{2}+(3 \lambda+1)\right)\right]}{9(1+\lambda)(1+2 \lambda)(1+3 \lambda)^{2}}\right] \\
& \times|\gamma|^{2}(1+3|\gamma|)+\frac{(1+4|\gamma|)(1+3|\gamma|)(3+8|\gamma|)|\gamma|^{2}}{6(1+2 \lambda)(1+4 \lambda)} .
\end{aligned}
$$

This completes the proof of this result.

\section{Conflict of Interests}

The authors declare that there is no conflict of interests regarding the publication of this paper.

\section{Acknowledgment}

The work here is supported by LRGS/TD/2011/UKM/ICT/ 03/02.

\section{References}

[1] H. Silverman, "Univalent functions with negative coefficients," Proceedings of the American Mathematical Society, vol. 51, pp. 109-116, 1975.

[2] M. Nunokawa, S. Owa, J. Nishiwaki, K. Kuroki, and T. Hayami, "Differential subordination and argumental property," Computers \& Mathematics with Applications, vol. 56, no. 10, pp. 193-217, 2008.

[3] J. W. Noonan and D. K. Thomas, "On the second Hankel determinant of areally mean p-valent functions," Transactions of the American Mathematical Society, vol. 223, pp. 337-346, 1976.

[4] M. Arif, K. I. Noor, and M. Raza, "Hankel determinant problem of a subclass of analytic functions," Journal of Inequalities and Applications, vol. 2012, article 22, 2012.

[5] M. Arif, K. I. Noor, M. Raza, and W. Haq, "Some properties of a generalized class of analytic functions related with Janowski functions," Abstract and Applied Analysis, vol. 2012, Article ID 279843, 11 pages, 2012.

[6] R. Ehrenborg, "The Hankel determinant of exponential polynomials," The American Mathematical Monthly, vol. 107, no. 6, pp. 557-560, 2000.

[7] J. W. Layman, "The Hankel transform and some of its properties," Journal of Integer Sequences, vol. 4, no. 1, pp. 1-11, 2001.
[8] A. Janteng, S. A. Halim, and M. Darus, "Hankel determinant for starlike and convex functions," International Journal of Mathematical Analysis, vol. 1, no. 13, pp. 619-625, 2007.

[9] K. O. Babalola, "On $\mathrm{H}_{3}$ (1) Hankel determinant for some classes of univalent functions," Inequality Theory and Applications, vol. 6, pp. 1-7, 2007.

[10] M. Raza and S. N. Malik, "Upper bound of the third Hankel determinant for a class of analytic functions related with lemniscate of Bernoulli," Journal of Inequalities and Applications, vol. 2013, article 412, 2013.

[11] C. Pommerenke, Univalent Functions, Vandenhoeck \& Ruprecht, Göttingen, Germany, 1975.

[12] F. R. Keogh and E. P. Merkes, "A coefficient inequality for certain classes of analytic functions," Proceedings of the American Mathematical Society, vol. 20, pp. 8-12, 1969.

[13] U. Grenander and G. Szegö, Toeplitz Forms and Their Applications, California Monographs in Mathematical Sciences, University of California, Berkeley, Calif, USA, 1958.

[14] J. Nishiwaki and S. Owa, "Coefficient inequalities for starlike and convex functions of reciprocal order," Electronic Journal of Mathematical Analysis and Applications, vol. 1, no. 2, pp. 212216, 2013 


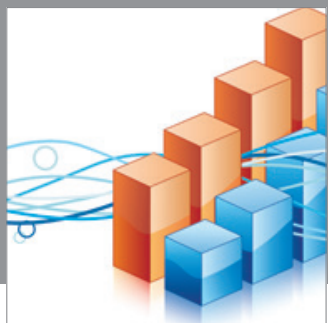

Advances in

Operations Research

mansans

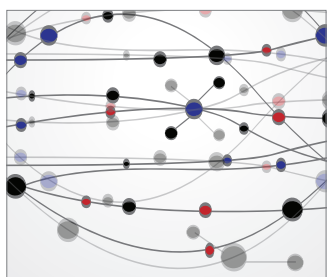

The Scientific World Journal
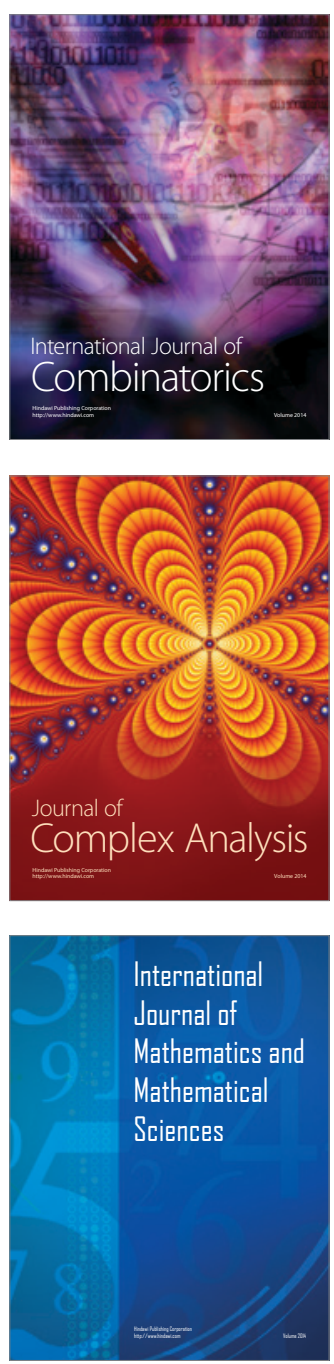
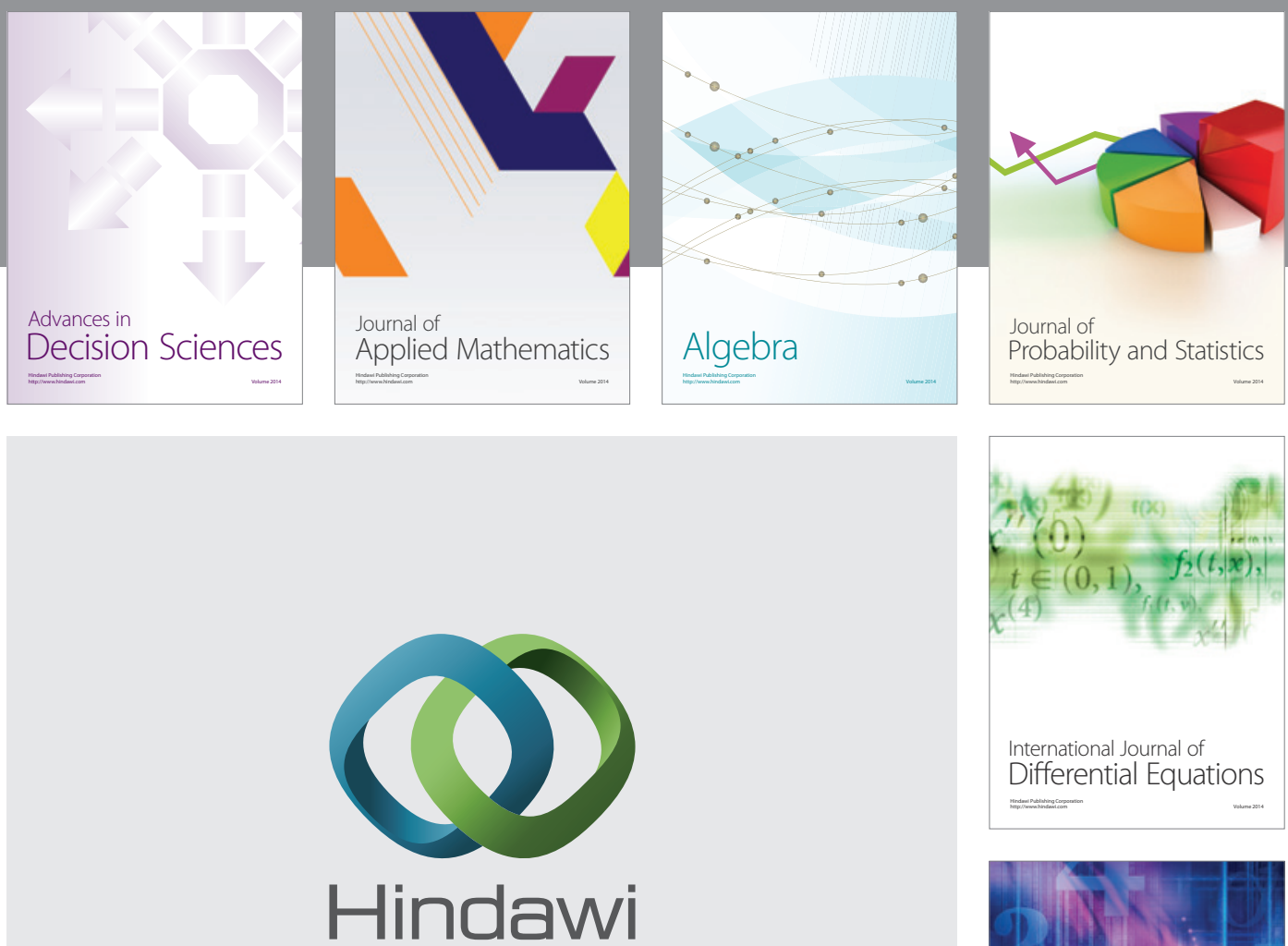

Submit your manuscripts at http://www.hindawi.com
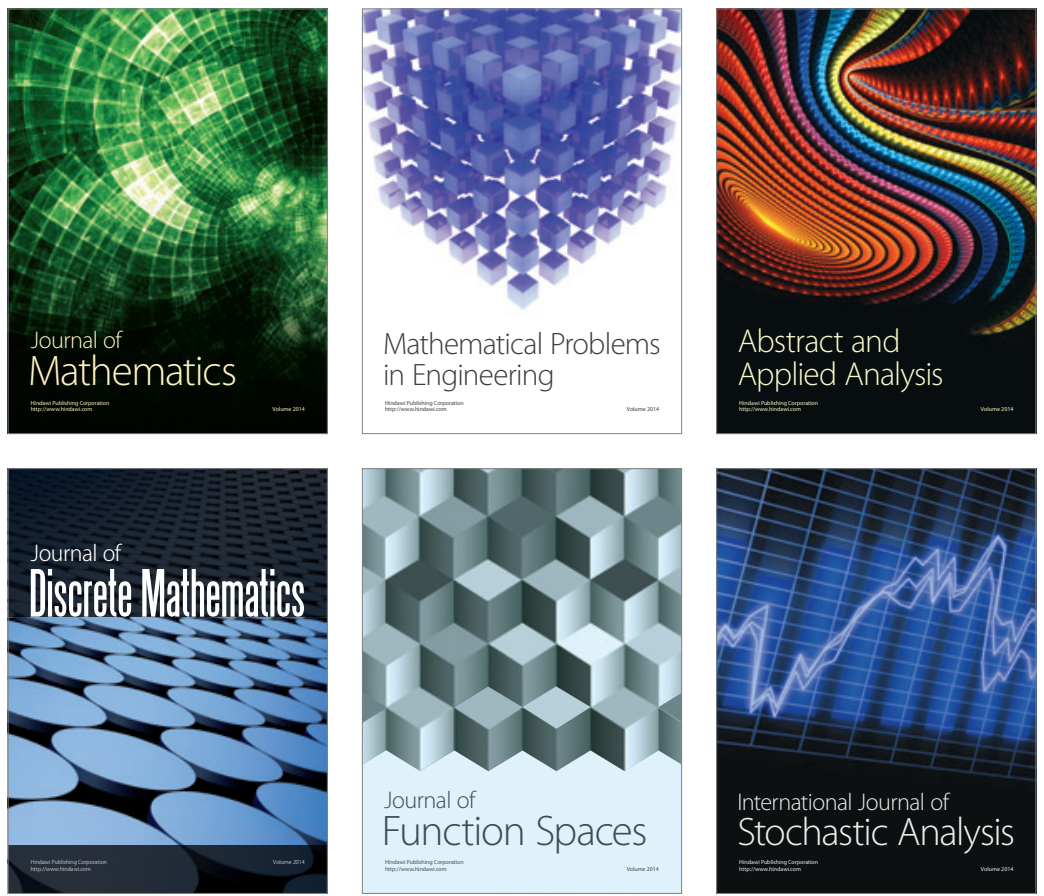

Journal of

Function Spaces

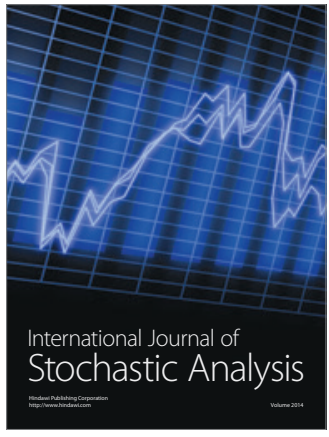

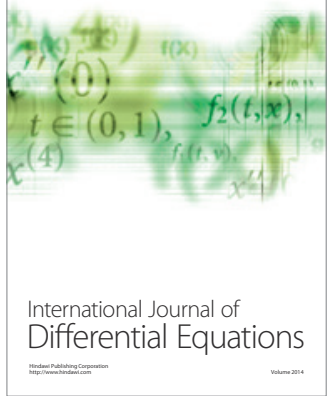
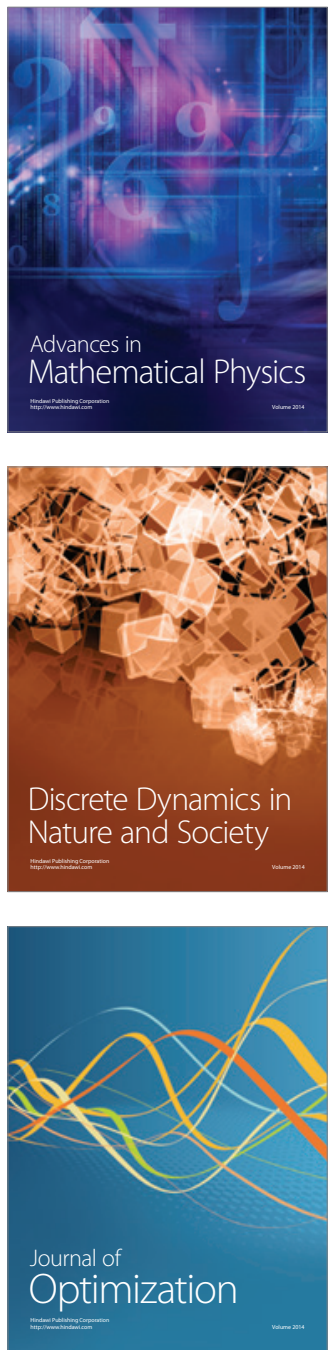